\title{
A. Perneczky, R. Reisch: Keyhole approaches in neurosurgery. Volume 1: Concept and surgical technique
}

\author{
Springer Wien, 2008, ISBN 978-3-211-83885-3
}

\author{
Nic de Tribolet
}

Received: 22 October 2009 / Accepted: 19 January 2011 /Published online: 11 February 2011

(C) Springer-Verlag 2011

This technical book describes the various classical microsurgical approaches using a small, so-called keyhole craniotomy.

There are eight chapters including an introduction. The introduction is a brief summary of the evolution of neurosurgical techniques towards microsurgery and endoscopy. The very small craniotomy implies an individually tailored approach and a predefined surgical corridor. These are addressed in detail in the subsequent chapters.

Each chapter starts with a historical review of the approach used followed by a description of the general anatomical construction of the region in question. A table summarizes the anatomical structures that can be approached by the craniotomy. The surgical technique itself starts with patient positioning followed by the craniotomy; each step is illustrated by a coloured drawing. Every step from the skin incision to the dura opening is detailed. Particular attention is given to the bone opening. The intradural dissection used to reach the area of interest is beautifully illustrated by microphotographs of coloured injected cadaveric preparations. There is a description of the dissection proceedure step by step accompanied with illustrations for each step. The closure is then described and potential errors are enumerated. Finally the chapter concludes with a section on tips and tricks illustrated with black and white drawings. These little technical details are very useful, for example, the direction of the burr hole trephination in the supraorbital craniotomy in order to avoid penetration of the orbit.

The chapters include: (1) an introduction; (2) the supraorbital approach with its lateral, medial and basal variarions; the latter includes fracturing of the orbital roof; (3) the subtemporal approach including a combined posterior subtemporal-presigmoidal variation and a trans-petrous-apex modification; (4) the retrosigmoidal approach; (5) the suboccipital approach; (6) the pineal approach; the infratentorial is descrbed here; (7) the interhemispheric approach, anterior and posterior; (8) the transcortical approach exposing the lateral and third ventricles. At the end, abundant references are quoted concerning history, microsurgical anatomy and operative approaches, imaging techniques and surgical planning, and finally surgical techniques, neuroendoscopy and operative traumatization.

Overall this is a good "cookbook". The reader will appreciate the excellent drawings of the various craniotomies and the photographs of the microsurgical anatomy. Although the information is not original, the size of the craniotomies is new and the presentation is didactic. This book will be of use for neurosurgeons who have some experience in microneurosurgery.

N. de Tribolet $(\triangle)$

Department of Neurosurgery,

University of Geneva Hospital (HUG),

1211 Geneva, Switzerland

e-mail: nicolas.detribolet@unige.ch 